\title{
A Mechanistically Guided Design Leads to the Synthesis of an Efficient and Practical New Reagent for the Highly Enantioselective, Catalytic Dihydroxylation of Olefins
}

\author{
Jinkun Huang and E. J. Corey* \\ Department of Chemistry and Chemical Biology \\ Harvard University, 12 Oxford Street, Cambridge, Massachusetts 02138.
}

\section{Supplementary Material}

General. Tetrahydrofuran, methylene chloride, toluene, N,N-dimethylformamide were dried using standard techniques and freshly distilled before use. When necessary, all reactions were carried out in oven $\left(120^{\circ} \mathrm{C}\right)$ or flame dried glassware under an atmosphere of nitrogen. Purification by flash column chromatography was performed on Baker 230-400 mesh silica gel. Melting points were determined using a Buchi hot oil bath apparatus and are uncorrected. Optical rotations were measured using a Perkin-Elmer 241 polarimeter. FTIR spectra were recorded as thin films on a Nicolet 5 ZDX FT-IR spectrometer. ${ }^{1} \mathrm{H}$ and ${ }^{13} \mathrm{C}$ NMR spectra were recorded on Varian Mercury and Inova series instruments $\left(400 \mathrm{MHz}\right.$ or $500 \mathrm{MHz}$ ) in $\mathrm{CDCl}_{3}$. Mass spectra analyses and high-resolution mass spectral analyses were measured on a JEOL model AX-505 or SX-102 spectrometer by means of electron ionization (EI), chemical ionization (CI), fast-atom bombardment (FAB) or electrospray (ESI). Chiralcel or Chiralpak HPLC columns were obtained from Daicel Chemical Industries, Ltd.

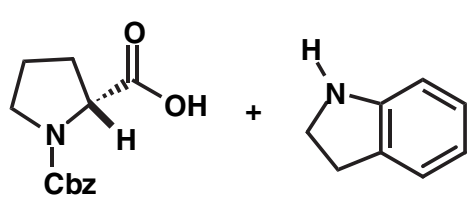

1. $(\mathrm{COCl})_{2}$

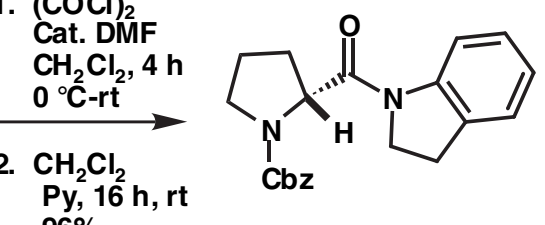

$\mathrm{Py}_{2}, 16 \mathrm{~h}, \mathrm{rt} \quad \mathrm{Cbz}$
$\mathrm{C}$ 
Coupling of Cbz-(R)-Proline with Dihydroindole. To the ice-cooled solution of Ncarbonylbenzyloxy-(R)-proline $(2.49 \mathrm{~g}, 10.0 \mathrm{mmol})$ and 5 drops of DMF in $\mathrm{CH}_{2} \mathrm{Cl}_{2}(30 \mathrm{~mL})$ was slowly added oxalic chloride $(1.50 \mathrm{~mL}, 17.1 \mathrm{mmol})$ by syringe in $20 \mathrm{~min}$. Evolution of gas was observed. The mixture was warmed to room temperature and stirred for another $5 \mathrm{~h}$. After concentration in vacuo $\mathrm{CH}_{2} \mathrm{Cl}_{2}(15 \mathrm{~mL})$ was added to the semi-solid residue and the resultant solution was cooled in an ice-bath and was treated with a solution of indoline (1.20 g, 10.0 mmol) and pyridine $(1.4 \mathrm{~mL}, 17.1 \mathrm{mmol})$ in $\mathrm{CH}_{2} \mathrm{Cl}_{2}(15 \mathrm{~mL})$. The mixture was stirred for $4 \mathrm{~h}$ at room temperature. The solution was successively washed with water, saturated aqueous sodium bicarbonate, hydrochloric acid $(1 \mathrm{~N})$ and brine. The organic layer was dried on $\mathrm{Na}_{2} \mathrm{SO}_{4}$. Removal of solvent provided an off-white solid as two rotamers: $3.48 \mathrm{~g}(99 \%) .[\alpha]_{\mathrm{D}}{ }^{22}=+31.2^{\circ}$ $\left(c=1.05, \mathrm{CHCl}_{3}\right) ;$ m.p. $137-138{ }^{\circ} \mathrm{C} .{ }^{1} \mathrm{H} \mathrm{NMR}\left(\mathrm{CDCl}_{3}, 500 \mathrm{MHz}\right): \delta 8.25(\mathrm{t}, J=9 \mathrm{~Hz}), 7.31-$ $7.38(\mathrm{~m}), 7.12-7.23(\mathrm{~m}), 6.98-7.07(\mathrm{~m}), 5.20(\mathrm{~d}, J=12 \mathrm{~Hz}), 5.08(\mathrm{~m}), 4.96(\mathrm{~d}, J=12 \mathrm{~Hz}), 4.67$ (m), $4.50(\mathrm{~m}), 4.42(\mathrm{~m}), 4.08(\mathrm{~m}), 3.85-3.92(\mathrm{~m}), 3.75(\mathrm{~m}), 3.56-3.64(\mathrm{~m}), 3.24(\mathrm{~m}), 3.06(\mathrm{~m})$, $2.83(\mathrm{~m}), 2.24(\mathrm{~m}), 2.05(\mathrm{~m}), 1.93(\mathrm{~m}) \mathrm{ppm} ;{ }^{13} \mathrm{C}\left(\mathrm{CDCl}_{3}, 400 \mathrm{~Hz}\right): \delta 171.13,170.77,155.17$, $154.32,143.24,143.13,136.98,136.43,131.30,128.68,128.46,128.24,128.14,128.08,128.04$, $127.84,127.74,124.67,124.15,124.11,117.67,117.57,67.65,67.26,59.19,58.64,47.8847 .74$, 47.50, 46.99, 30.83, 29.84, 28.42, 28.15, 24.60, 24.04 ppm. FTIR (cm ${ }^{-1}$, film): 2969, 2883, 1687, 1667, 1655, 1478, 1415, 1360, 1337, 1301, 1128, 731. MS (CI) m/z: 351([M + $\left.\left.\mathrm{H}^{+}\right]\right)$. HRMS calcd for $\left[\mathrm{C}_{21} \mathrm{H}_{22} \mathrm{~N}_{2} \mathrm{O}_{3}+\mathrm{H}^{+}\right]$: 351.1708 , found: 351.1702 .

Its enantiomer was synthesized from $\mathrm{N}$-carbonylbenzyloxy-(S)-proline in the same manner and is physically identical except for $[\alpha]_{\mathrm{D}}^{22}=-32.2^{\circ}\left(c=1.10, \mathrm{CHCl}_{3}\right)$.

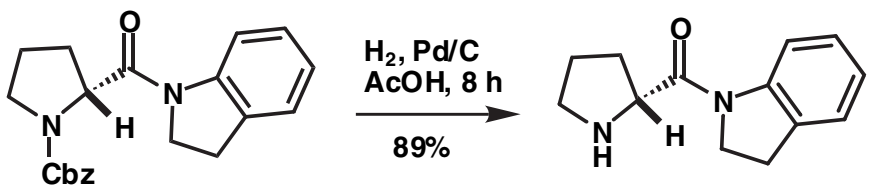


Compound 9. The above indoline amide (4.20 g, $12.0 \mathrm{mmol})$ was dissolved in acetic acid (50 $\mathrm{mL})$ and added to a flask charged with $\mathrm{Pd} / \mathrm{C}(10 \% \mathrm{wt}, 1.3 \mathrm{~g})$. The mixture was degassed and refilled with hydrogen via a balloon and this process was repeated five times. The mixture was stirred overnight under a hydrogen atmosphere. Removal of the catalyst and concentration gave 9 as a brown oil which was purified by flash chromatography $\left(\mathrm{MeOH} / \mathrm{CH}_{2} \mathrm{Cl}_{2} / \mathrm{NH}_{4} \mathrm{OH}=\right.$ $5 / 95 / 0.5, \mathrm{Rf}=0.21): \tan$ oil, $2.55 \mathrm{~g}(89 \%) . \quad[\alpha]_{\mathrm{D}}{ }^{25}=+104.6^{\circ}\left(c=1.25, \mathrm{CHCl}_{3}\right) .{ }^{1} \mathrm{H} \mathrm{NMR}$ $\left(\mathrm{CDCl}_{3}, 500 \mathrm{MHz}\right): \delta 8.22(\mathrm{~d}, J=8.5 \mathrm{~Hz}, 1 \mathrm{H}), 7.21(\mathrm{~m}, 2 \mathrm{H}), 7.04(\mathrm{t}, J=7.0 \mathrm{~Hz}, 1 \mathrm{H}), 4.16(\mathrm{~m}$, $1 \mathrm{H}), 4.09(\mathrm{~m}, 1 \mathrm{H}), 3.94(\mathrm{~m}, 1 \mathrm{H}), 3.24(\mathrm{~m}, 3 \mathrm{H}), 2.91(\mathrm{~m}, 1 \mathrm{H}), 2.72$ (br, $1 \mathrm{H}), 2.22(\mathrm{~m}, 1 \mathrm{H})$, $1.78-1.87(\mathrm{~m}, 3 \mathrm{H}) \mathrm{ppm} ;{ }^{13} \mathrm{C}\left(\mathrm{CDCl}_{3}, 400 \mathrm{~Hz}\right): \delta 172.84,143.20,131.31,127.85,124.83$, 124.22, 117.39, 60.57, 48.12, 47.58, 30.83, 28.41, 26.78 ppm. FTIR ( $\mathrm{cm}^{-1}$, film): 3296, 2951, 2861, 1649, 1598, 1480, 1461, 1392, 1289, 1263, 752. MS (CI) $m / z: 216\left(\left[\mathrm{M}+\mathrm{H}^{+}\right]\right)$. HRMS calcd for $\left[\mathrm{C}_{13} \mathrm{H}_{16} \mathrm{~N}_{2} \mathrm{O}+\mathrm{H}^{+}\right]$: 216.1341, found: 216.1346 .

Its enantiomer was synthesized in the same manner and is identical to 9 except for $[\alpha]_{\mathrm{D}}{ }^{23}$ $=-107.8^{\circ}\left(c=1.0, \mathrm{CHCl}_{3}\right)$

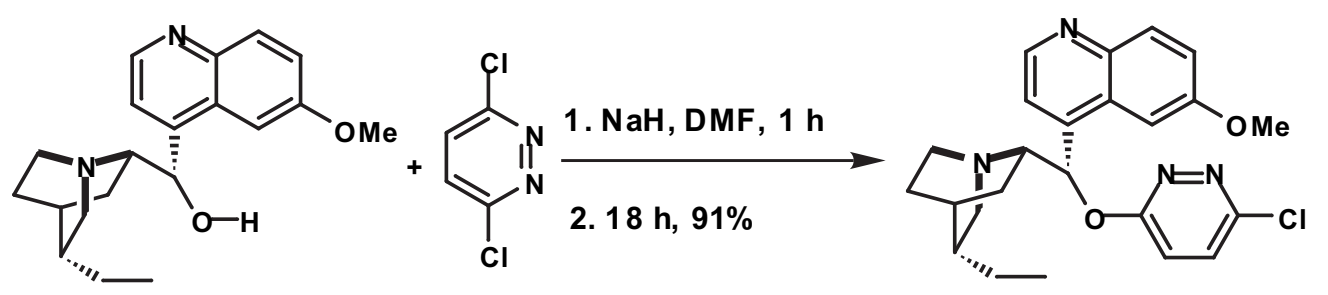

Compound 8. Sodium hydride (57-63\% in mineral oil, $120 \mathrm{mg}, 3.0 \mathrm{mmol}$ ) and dimethyl formamide $(5 \mathrm{~mL})$ were charged into a flame-dried flask under a nitrogen atmosphere at $10{ }^{\circ} \mathrm{C}$. A solution of hydroquinidine $(326 \mathrm{mg}, 1.0 \mathrm{mmol})$ in dimethyl formamide $(3 \mathrm{~mL})$ was slowly added to the vigorously stirred suspension by syringe. The mixture was stirred at $23{ }^{\circ} \mathrm{C}$ for another $3 \mathrm{~h}$ followed by addition of solution of 3,6-dichloropyridazine (150 $\mathrm{mg}, 1.0 \mathrm{mmol})$ in dimethyl formamide $(2 \mathrm{~mL})$. The resultant mixture was stirred at $23{ }^{\circ} \mathrm{C}$ overnight. The solvent was removed by aspirator and the residue was extracted with diethyl ether $(3 \times 20 \mathrm{~mL})$. The organic extracts were washed with brine and dried on $\mathrm{Na}_{2} \mathrm{SO}_{4}$. The solvent was removed and the 
residue was purified by flash chromatography $(2 \%$ triethyl amine in ethyl acetate, $\mathrm{Rf}=0.15)$ : colorless solid; $400 \mathrm{mg}, 91 \%$. $[\alpha]_{\mathrm{D}}^{22}=-41.4^{\circ}\left(c=1.0, \mathrm{CHCl}_{3}\right)$. m.p.: $73-74{ }^{\circ} \mathrm{C} .{ }^{1} \mathrm{H}$ NMR $\left(\mathrm{CDCl}_{3}, 500 \mathrm{MHz}\right): \delta 8.68(\mathrm{~d}, J=5 \mathrm{~Hz}, 1 \mathrm{H}), 8.00(\mathrm{~d}, J=9 \mathrm{~Hz}, 1 \mathrm{H}), 7.52(\mathrm{~s}, 1 \mathrm{H}), 7.36(\mathrm{~m}, 3$ H), $7.05(\mathrm{~d}, J=9.5 \mathrm{~Hz}, 1 \mathrm{H}), 3.98(\mathrm{~s}, 3 \mathrm{H}), 3.39$ (d, $J=7 \mathrm{~Hz}, 1 \mathrm{H}), 2.75-2.96(\mathrm{~m}, 5 \mathrm{H}), 1.95$ (m, $1 \mathrm{H}), 1.77(\mathrm{~m}, 1 \mathrm{H}), 1.49-1.63(\mathrm{~m}, 6 \mathrm{H}), 0.93(\mathrm{t}, J=6 \mathrm{~Hz}, 3 \mathrm{H}) \mathrm{ppm} ;{ }^{13} \mathrm{C}\left(\mathrm{CDCl}_{3}, 400 \mathrm{~Hz}\right): \delta$ $163.58,158.14,151.63,147.55,144.98,131.98,131.3,127.42,122.13,120.12,118.82,101.86$, $59.95,55.92,51.26,50.33,49.31,45.98,45.37,44.63,42.28,38.74,37.55,34.79,31.30,30.91$, 28.47, 27.44, 26.26, 25.71, 23.48, 12.24 ppm. FTIR ( $\mathrm{cm}^{-1}$, film): 2930, 2863, 1620, 1581, 1502, 1411, 1293, 1223, 1022, 983, 825, 719. MS (CI) $m / z: 439\left(\left[\mathrm{M}+\mathrm{H}^{+}\right]\right)$. HRMS calcd for $\left[\mathrm{C}_{24} \mathrm{H}_{27} \mathrm{ClN}_{4} \mathrm{O}_{2}+\mathrm{H}^{+}\right]:$439.1901, found: 439.1911 .

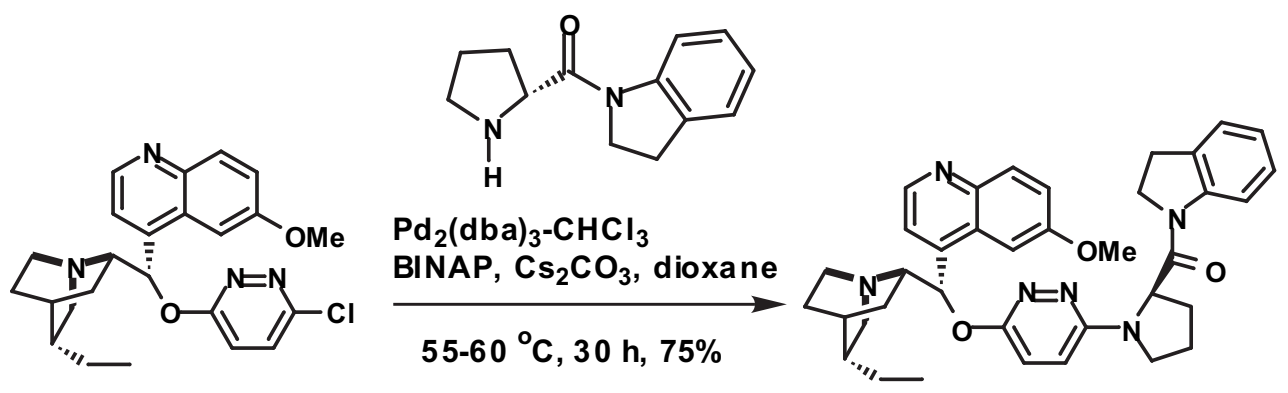

Compound 4. Compound 9 (207 mg, $0.96 \mathrm{mmol}$ ), Compound 8 (420 mg, $0.96 \mathrm{mmol}$ ), $\mathrm{Pd}_{2}(\mathrm{dba})_{3} \cdot \mathrm{CHCl}_{3}(50 \mathrm{mg}, 0.05 \mathrm{mmol})$, racemic-BINAP (62 mg, $\left.0.10 \mathrm{mmol}\right), \mathrm{Cs}_{2} \mathrm{CO}_{3}(938 \mathrm{mg}$, $2.88 \mathrm{mmol})$ and dioxane $(2 \mathrm{~mL})$ were mixed in a flame-dried test tube. The mixture was degassed, refilled with nitrogen and stirred at $55-60{ }^{\circ} \mathrm{C}$ for $30 \mathrm{~h}$. The mixture was cooled to room temperature and poured into ethyl acetate $(50 \mathrm{~mL})$. The organics was successively washed with water, brine and dried on sodium sulfate. The solvent was evaporated under vacuum and the residue was purified by flash (1-2\% triethyl amine in ethyl acetate, Rf $=0.1-0.15)$ chromatography: colorless solid, $415 \mathrm{mg}, 75 \%$. $[\alpha]_{\mathrm{D}}^{22}=+29.0^{\circ}\left(c=1.2, \mathrm{CHCl}_{3}\right)$. m.p.: 131$132{ }^{\circ} \mathrm{C} .{ }^{1} \mathrm{H} \mathrm{NMR}\left(\mathrm{CDCl}_{3}, 400 \mathrm{MHz}\right): \delta 8.64(\mathrm{~d}, J=4 \mathrm{~Hz}, 1 \mathrm{H}), 8.16(\mathrm{~d}, J=8 \mathrm{~Hz}, 1 \mathrm{H}), 7.95$ $(\mathrm{d}, J=9 \mathrm{~Hz}, 1 \mathrm{H}), 7.53(\mathrm{~s}, 1 \mathrm{H}), 7.40(\mathrm{~d}, J=4 \mathrm{~Hz}, 1 \mathrm{H}), 7.32$ (dd, $J=9 \mathrm{~Hz}, J=2 \mathrm{~Hz}, 1 \mathrm{H}), 7.20$ 
$(\mathrm{d}, J=7 \mathrm{~Hz}, 1 \mathrm{H}), 7.15(\mathrm{t}, J=7 \mathrm{~Hz}, 1 \mathrm{H}), 7.01(\mathrm{t}, J=7 \mathrm{~Hz}, 1 \mathrm{H}), 6.89(\mathrm{~d}, J=9 \mathrm{~Hz}, 1 \mathrm{H}), 6.75$ (m, $2 \mathrm{H}), 4.86(\mathrm{~d}, J=5 \mathrm{~Hz}, 1 \mathrm{H}), 4.44(\mathrm{~m}, 1 \mathrm{H}), 4.04(\mathrm{~m}, 1 \mathrm{H}), 3.87$ (s, $3 \mathrm{H}), 3.69$ (m, $1 \mathrm{H}), 3.43$ $(\mathrm{m}, J=8 \mathrm{~Hz}, 1 \mathrm{H}), 3.17-3.30(\mathrm{~m}, 3 \mathrm{H}), 2.71-2.82(\mathrm{~m}, 4 \mathrm{H}), 2.28(\mathrm{~m}, 2 \mathrm{H}), 2.06(\mathrm{~m}, 2 \mathrm{H}), 1.94$ $(\mathrm{m}, 1 \mathrm{H}), 1.70(\mathrm{~m}, 1 \mathrm{H}), 1.44-1.51(\mathrm{~m}, 5 \mathrm{H}), 0.85(\mathrm{t}, J=7 \mathrm{~Hz}, 3 \mathrm{H}) \mathrm{ppm} ;{ }^{13} \mathrm{C}\left(\mathrm{CDCl}_{3}, 400 \mathrm{~Hz}\right): \delta$ $171.68,158.35,155.03,147.69,144.90,143.55,131.73,131.38,127.66,124.72,123.90,121.80$, $119.39,119.01,117.47,117.36,102.49,60.46,59.57,55.86,51.09,50.26,47.95,47.78,37.78$, 30.08, 28.44, 27.72, 26.38, 25.55, 24.06, 23.58, 12.22 ppm. FTIR ( $\mathrm{cm}^{-1}$, film): 2936, 2865, 1666, 1456, 1427, 1288, 1213, 988, 831, 749. MS (CI) $m / z: 619\left(\left[\mathrm{M}+\mathrm{H}^{+}\right]\right)$. HRMS calcd for $\left[\mathrm{C}_{37} \mathrm{H}_{42} \mathrm{~N}_{4} \mathrm{O}_{3}+\mathrm{H}^{+}\right]:$619.3396, found: 619.3386 .

The (S)-proline analogue of compound 4 (5) was synthesized in the same manner as described above: colorless solid, $62 \%$; $[\alpha]_{\mathrm{D}}^{22}=-4.2^{\circ}\left(c=0.95, \mathrm{CHCl}_{3}\right)$. m.p.: $144-145{ }^{\circ} \mathrm{C} .{ }^{1} \mathrm{H}$ NMR $\left(\mathrm{CDCl}_{3}, 400 \mathrm{MHz}\right): \delta 8.64(\mathrm{~d}, J=4 \mathrm{~Hz}, 1 \mathrm{H}), 8.09(\mathrm{~d}, J=8 \mathrm{~Hz}, 1 \mathrm{H}), 7.93(\mathrm{~d}, J=9 \mathrm{~Hz}, 1$ H), $7.47(\mathrm{~s}, 1 \mathrm{H}), 7.39$ (d, $J=4 \mathrm{~Hz}, 1 \mathrm{H}), 7.28(\mathrm{dd}, J=9 \mathrm{~Hz}, J=2 \mathrm{~Hz}, 1 \mathrm{H}), 7.16(\mathrm{~d}, J=7 \mathrm{~Hz}, 1$ H), $7.12(\mathrm{t}, J=7 \mathrm{~Hz}, 1 \mathrm{H}), 6.98(\mathrm{t}, J=7 \mathrm{~Hz}, 1 \mathrm{H}), 6.88(\mathrm{~m}, 2 \mathrm{H}), 6.72(\mathrm{~d}, J=9 \mathrm{~Hz}, 1 \mathrm{H}), 4.89$ (d, $J=5 \mathrm{~Hz}, 1 \mathrm{H}), 4.50(\mathrm{~m}, 1 \mathrm{H}), 4.09(\mathrm{~m}, 1 \mathrm{H}), 3.84(\mathrm{~s}, 3 \mathrm{H}), 3.74(\mathrm{~m}, 1 \mathrm{H}), 3.50(\mathrm{~m}, J=8 \mathrm{~Hz}, 1$ H), $3.36(\mathrm{~m}, 1 \mathrm{H}), 3.20(\mathrm{~m}, 2 \mathrm{H}), 2.75-2.85(\mathrm{~m}, 4 \mathrm{H}), 2.31(\mathrm{~m}, 2 \mathrm{H}), 2.06(\mathrm{~m}, 3 \mathrm{H}), 1.71(\mathrm{~m}, 1 \mathrm{H})$, 1.25-1.50 (m, $5 \mathrm{H}), 0.89(\mathrm{t}, J=7 \mathrm{~Hz}, 3 \mathrm{H}) \mathrm{ppm} ;{ }^{13} \mathrm{C}\left(\mathrm{CDCl}_{3}, 400 \mathrm{~Hz}\right): \delta 171.34,158.66,155.07$, $147.72,144.90,143.40,131.82,131.20,127.73,124.72,123.97,121.78,119.41,119.06,117.43$, $102.39,59.62,55.86,51.00,50.26,48.02,47.76,37.77,30.25,28.47,27.63,26.41,25.56,23.98$, 22.88, 12.30 ppm. FTIR (cm ${ }^{-1}$, film): 2926, 2867, 1659, 1616, 1451, 1411, 1286, 1223, 1022 , 987, 821, 754. MS (CI) $m / z: 619\left(\left[\mathrm{M}+\mathrm{H}^{+}\right]\right)$. HRMS calcd for $\left[\mathrm{C}_{37} \mathrm{H}_{42} \mathrm{~N}_{4} \mathrm{O}_{3}+\mathrm{H}^{+}\right]: 619.3396$, found: 619.3398 .

General Procedure for Catalytic Asymmetric Dihydroxylation. A mixture of ligand 4 (5 mg, $0.0081 \mathrm{mmol}), \mathrm{K}_{2} \mathrm{OsO}_{4} \cdot 2 \mathrm{H}_{2} \mathrm{O}(1.0 \mathrm{mg}, 0.0027 \mathrm{mmol}), \mathrm{K}_{3} \mathrm{Fe}(\mathrm{CN})_{6}(1.18 \mathrm{~g}, 3.57 \mathrm{mmol}), \mathrm{K}_{2} \mathrm{CO}_{3}$ (0.493 g, $3.57 \mathrm{mmol})$ and $\mathrm{MeSO}_{2} \mathrm{NH}_{2}(113 \mathrm{mg}, 1.19 \mathrm{mmol}$, only when 1,2-disubstituted or tri- 
substituted olefins were used as substrates) in $12 \mathrm{~mL}$ of $1: 1 t-\mathrm{BuOH}-\mathrm{H}_{2} \mathrm{O}$ was stirred at $0{ }^{\circ} \mathrm{C}$ for $0.5 \mathrm{~h}$. The olefin $(1.19 \mathrm{mmol})$ was added to the suspension and the resulting mixture was stirred vigorously at $0{ }^{\circ} \mathrm{C}$ for $8-24 \mathrm{~h}$. The reaction was monitored by TLC (20-50\% EtOAc/hexane). After completion of the reaction as indicated by TLC, the mixture was quenched with $\mathrm{Na}_{2} \mathrm{SO}_{3}$ $(1.5 \mathrm{~g})$ at $0{ }^{\circ} \mathrm{C}$, and was then warmed to room temperature and stirred for $45 \mathrm{~min}$. The reaction mixture was extracted three times with ethyl acetate $(3 \times 25 \mathrm{~mL})$. The organic extract was washed with $1 \mathrm{~N} \mathrm{KOH}$ (to remove methyl sulfonamide when necessary) and $15 \mathrm{~mL}$ of brine, dried over $\mathrm{Na}_{2} \mathrm{SO}_{4}$, and concentrated in vacuo. The residue was purified by silica gel chromatography to give the diol (10-70\% EtOAc in hexane) as colorless solid or colorless oil and the recovered ligand 4 (5\% TEA/EtOAc). The enantiomeric excess (ee) of the diols was determined either by chiral-column HPLC analysis or ${ }^{1} \mathrm{H}$ NMR analysis of the corresponding MTPA esters.

\section{Characterization of Diols.}<smiles>C=Cc1ccccc1</smiles>

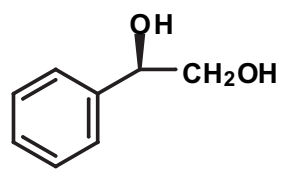

Rotation: $[\alpha]_{\mathrm{D}}^{23}[\mathrm{c}=1.1,95 \% \mathrm{EtOH}]-36.7$. The enantiomeric purity was determined by ${ }^{1} \mathrm{H}$ NMR integration of the corresponding bis-MTPA ester derivative $\left(\mathrm{CDCl}_{3}, 500 \mathrm{MHz}\right): \delta 6.28$ ppm (dd, $1 \mathrm{H},\left(\mathrm{R}\right.$, major)), $6.18 \mathrm{ppm}(\mathrm{dd}, 1 \mathrm{H},(\mathrm{S}, \text { minor }))^{1}$

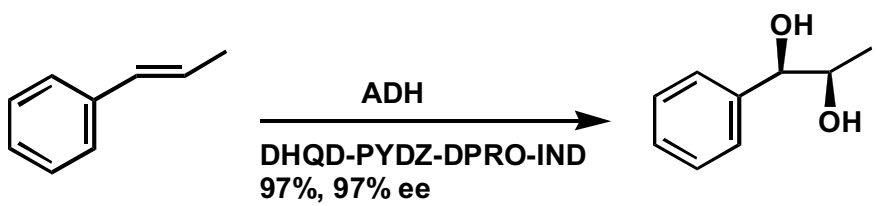


Rotation: $[\alpha]^{23}[\mathrm{c}=1.1,95 \% \mathrm{EtOH}]-31.3$. The enantiomeric purity was determined by ${ }^{1} \mathrm{H}$ NMR integration of the corresponding bis-MTPA ester derivative $\left(\mathrm{CDCl}_{3}, 500 \mathrm{MHz}\right): \delta 5.97$ ppm (d, $1 \mathrm{H},(\mathrm{R}$, major $)), 6.03 \mathrm{ppm}(\mathrm{d}, 1 \mathrm{H},(\mathrm{S}, \text { minor }))^{2}$
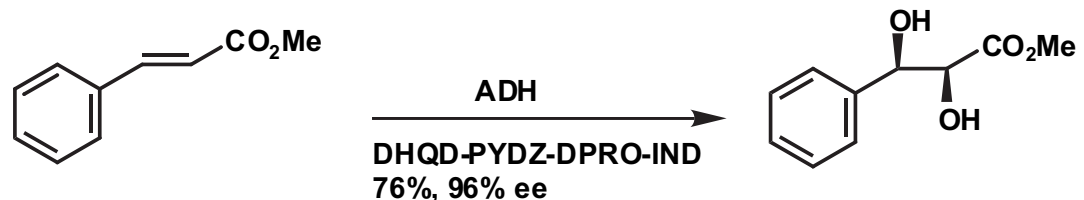

Rotation: $[\alpha]_{\mathrm{D}}^{23}[\mathrm{c}=1.1,95 \% \mathrm{EtOH}]-4.55$. The enantiomeric purity was determined by ${ }^{1} \mathrm{H}$ NMR integration of the corresponding bis-MTPA ester derivative $\left(\mathrm{CDCl}_{3}, 500 \mathrm{MHz}\right): \delta 5.51$ $\operatorname{ppm}(\mathrm{d}, 1 \mathrm{H},(1 \mathrm{R}, 2 \mathrm{~S}$ major $)), 5.46 \mathrm{ppm}(\mathrm{d}, 1 \mathrm{H},(1 \mathrm{~S}, 2 \mathrm{R}, \text { minor }))^{3}$<smiles>C(=C/c1ccccc1)\c1ccccc1</smiles>
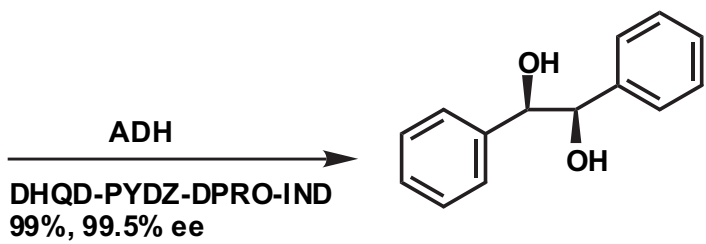

Rotation: $[\alpha]^{23}[\mathrm{c}=1.0,95 \% \mathrm{EtOH}]+92.6$. The enantiomeric purity was determined by ${ }^{1} \mathrm{H}$ NMR integration of the corresponding bis-MTPA ester derivative $\left(\mathrm{CDCl}_{3}, 500 \mathrm{MHz}\right): \delta$ $6.21 \mathrm{ppm}(\mathrm{s}, 1 \mathrm{H},(\mathrm{R}, \mathrm{R}$, major $)), 6.32 \mathrm{ppm}(\mathrm{s}, 1 \mathrm{H},(\mathrm{S}, \mathrm{S}$ minor $)){ }^{4}$
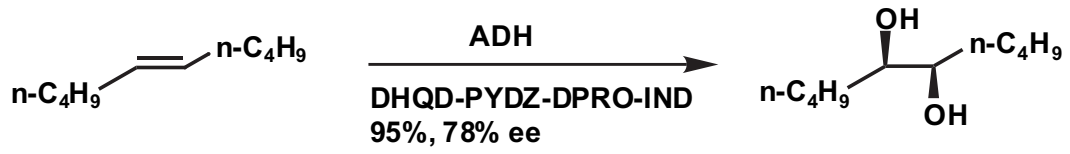

Rotation: $[\alpha]_{\mathrm{D}}^{24}[\mathrm{c}=1.1,95 \% \mathrm{EtOH}]+30.5$. The enantiomeric purity was determined by ${ }^{1} \mathrm{H}$ NMR integration of the corresponding bis-MTPA ester derivative $\left(\mathrm{CDCl}_{3}, 400 \mathrm{MHz}\right): \delta$ $5.22 \mathrm{ppm}(\mathrm{t}, J=6.4 \mathrm{~Hz}, 1 \mathrm{H},(\mathrm{R}, \mathrm{R}$, major $)), 5.13 \mathrm{ppm}(\mathrm{t}, J=6.4 \mathrm{~Hz}, 1 \mathrm{H},(\mathrm{S}, \mathrm{S} \text { minor }))^{5}$ 


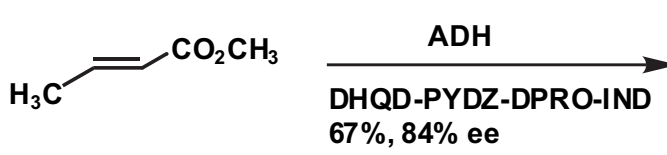

$\overbrace{\mathrm{OH}}^{\mathrm{OH}} \mathrm{CO}_{2} \mathrm{CH}_{3}$

Rotation: $[\alpha]^{23}[\mathrm{c}=1.0,95 \% \mathrm{EtOH}]-3.6$. The enantiomeric purity was determined by ${ }^{1} \mathrm{H}$ NMR integration of the corresponding bis-MTPA ester derivative $\left(\mathrm{CDCl}_{3}, 500 \mathrm{MHz}\right): \delta 5.70$ $\operatorname{ppm}(\mathrm{qd}, J=6.0 \mathrm{~Hz}, J=2.0 \mathrm{~Hz}, 1 \mathrm{H},(2 \mathrm{~S}, 3 \mathrm{R}$, major $)), 5.62 \mathrm{ppm}(\mathrm{qd}, J=6.0 \mathrm{~Hz}, J=2.0 \mathrm{~Hz}, 1 \mathrm{H}$, (2R,3S minor)). ${ }^{6}$
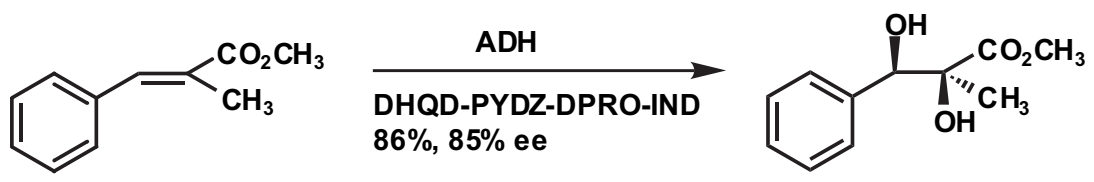

Rotation: $[\alpha]_{\mathrm{D}}^{23}\left[\mathrm{c}=1.4, \mathrm{CHCl}_{3}\right]-8.0$. The enantiomeric purity was determined by ${ }^{1} \mathrm{H}$ NMR integration of the corresponding mono-MTPA ester derivative $\left(\mathrm{CDCl}_{3}, 500 \mathrm{MHz}\right): \delta 6.02$ ppm (s, $1 \mathrm{H},\left(2 \mathrm{~S}, 3 \mathrm{R}\right.$, major)), $6.05 \mathrm{ppm}(\mathrm{s}, 1 \mathrm{H},(2 \mathrm{R}, 3 \mathrm{~S}, \text { minor }))^{7}$<smiles>C1=C(c2ccccc2)CCCC1</smiles>
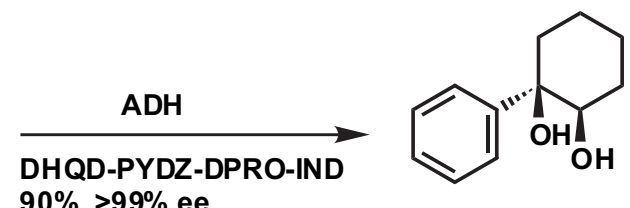

Rotation: $[\alpha]^{23}[\mathrm{c}=1.2,95 \% \mathrm{EtOH}]-7.1$ or $[\mathrm{c}=1.0$, benzene $]+17.6$. The enantiomeric purity was determined by chiral HPLC analysis (2\% 2-propanol in hexane, Chiralcel OJ column, $1 \mathrm{~mL} / \mathrm{min}$ flow rate, $\lambda=254 \mathrm{~nm}$, retention times: $37.6 \mathrm{~min}(\mathrm{R}, \mathrm{R}$, major), $26.7 \mathrm{~min}(\mathrm{~S}, \mathrm{~S}$, minor $)){ }^{8}$

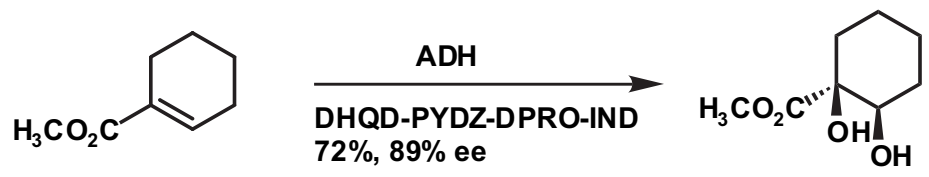


Rotation: $[\alpha]^{24}[\mathrm{c}=1.1,95 \% \mathrm{EtOH}]+2.5 .{ }^{1} \mathrm{H} \mathrm{NMR}\left(\mathrm{CDCl}_{3}, 400 \mathrm{MHz}\right): \delta .81(\mathrm{~m}, 1 \mathrm{H})$, $3.80(\mathrm{~s}, 3 \mathrm{H}), 1.67-1.85(\mathrm{~m}, 4 \mathrm{H}), 1.48-1.56(\mathrm{~m}, 3 \mathrm{H}), 1.32(\mathrm{~m}, 1 \mathrm{H}) \mathrm{ppm} .{ }^{13} \mathrm{C}\left(\mathrm{CDCl}_{3}, 400 \mathrm{~Hz}\right)$ : $\delta 176.92,77.02,72.47,53.28,34.59,30.52,24.27,20.09$ ppm. FTIR $\left(\mathrm{cm}^{-1}\right.$, film): 3465,2933 , 2852, 1728, 1438, 1238, 1148, 1066, 1029, 997. MS (CI): $192\left(\mathrm{M}+\mathrm{NH}_{4}{ }^{+}\right)$. Calculated for $\left(\mathrm{C}_{8} \mathrm{H}_{14} \mathrm{O}_{4}+\mathrm{NH}_{4}^{+}\right)$: 192.1236 ; Found: 192.1240 . The enantiomeric purity was determined by ${ }^{1} \mathrm{H}$ NMR integration of the corresponding mono-MTPA ester derivative $\left(\mathrm{CDCl}_{3}, 500 \mathrm{MHz}\right): \delta 3.47$ ppm (s, $1 \mathrm{H},(1 \mathrm{R}, 2 \mathrm{R}$, major)), $3.51 \mathrm{ppm}(\mathrm{s}, 1 \mathrm{H},(1 \mathrm{~S}, 2 \mathrm{~S}$ minor)).
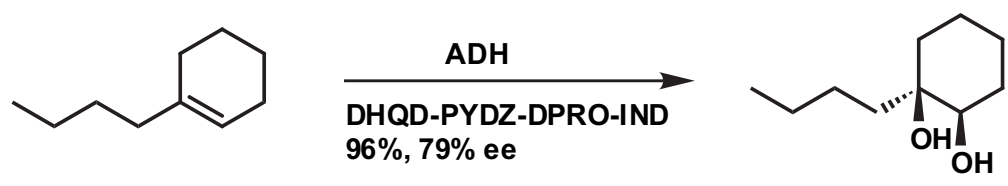

Rotation: $[\alpha]_{\mathrm{D}}^{24}\left[\mathrm{c}=1.0, \mathrm{CHCl}_{3}\right]+3.2 .{ }^{1} \mathrm{H} \mathrm{NMR}\left(\mathrm{CDCl}_{3}, 400 \mathrm{MHz}\right): \delta 3.45(\mathrm{~m}, 1 \mathrm{H})$, $1.76-1.29(\mathrm{~m}, 14 \mathrm{H}), 0.91(\mathrm{t}, J=6.4 \mathrm{~Hz}, 3 \mathrm{H}) \mathrm{ppm} .{ }^{13} \mathrm{C}\left(\mathrm{CDCl}_{3}, 400 \mathrm{~Hz}\right): \delta 73.61,73.58,38.71$, 33.78, 30.58, 25.63, 23.59, 23.31, 21.50, 14.34 ppm. FTIR $\left(\mathrm{cm}^{-1}\right.$, film): 3322, 2937, 2854, 1447, 1073, 975. m.p. 84-86 ${ }^{\circ} \mathrm{C}$. $\mathrm{MS}(\mathrm{EI})$ : 172. Calculated for $\mathrm{C}_{10} \mathrm{H}_{20} \mathrm{O}_{2}$ : 172.1463; Found: 172.1468. The enantiomeric purity was determined by ${ }^{1} \mathrm{H}$ NMR integration of the corresponding mono-MTPA ester derivative $\left(\mathrm{CDCl}_{3}, 500 \mathrm{MHz}\right): \delta 3.49 \mathrm{ppm}(\mathrm{s}, 1 \mathrm{H},(1 \mathrm{~S}, 2 \mathrm{R}$, major $)), 3.55 \mathrm{ppm}$ (s, $1 \mathrm{H},(1 \mathrm{R}, 2 \mathrm{~S}$, minor $))^{9}$
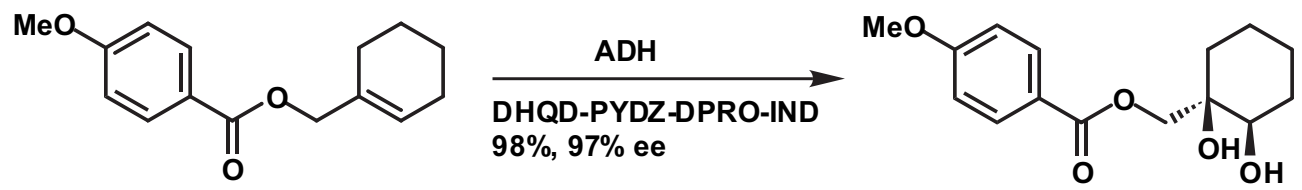

Rotation: $[\alpha]^{23}\left[\mathrm{c}=1.3, \mathrm{CHCl}_{3}\right]+4.7$. The enantiomeric purity was determined by chiral HPLC analysis (10\% 2-propanol in hexane, Chiralcel OD column, $1 \mathrm{~mL} / \mathrm{min}$ flow rate, $\lambda=$ $254 \mathrm{~nm}$, retention times: $43.7 \mathrm{~min}(1 \mathrm{~S}, 2 \mathrm{R}$, major), $33.4 \mathrm{~min}(1 \mathrm{R}, 2 \mathrm{~S}$, minor $)) .{ }^{10}$ 


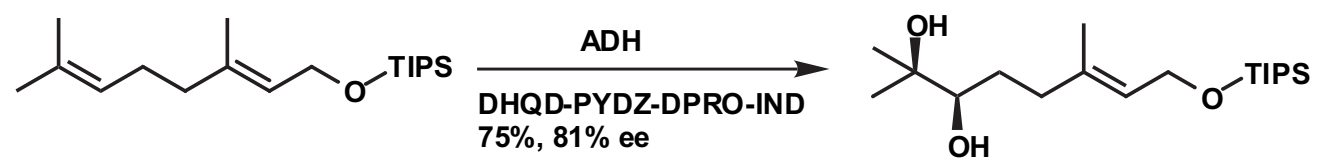

Rotation: $[\alpha]^{23}[\mathrm{c}=2.0,95 \% \mathrm{EtOH}]+15.7 .{ }^{1} \mathrm{H} \mathrm{NMR}\left(\mathrm{CDCl}_{3}, 500 \mathrm{MHz}\right): \delta 5.41(\mathrm{dt}, 1$ $\mathrm{H}, \mathrm{J}=1.5,7.2 \mathrm{~Hz}), 4.25(\mathrm{dd}, 2 \mathrm{H}, \mathrm{J}=1.0,6.5 \mathrm{~Hz}), 3.87(\mathrm{dd}, 1 \mathrm{H}, \mathrm{J}=5.5,2 \mathrm{~Hz}) 2.28(\mathrm{~m}, 1 \mathrm{H})$, $2.10(\mathrm{~m}, 1 \mathrm{H}), 1.80(\mathrm{br}, 2 \mathrm{H}), 1.64(\mathrm{~s}, 3 \mathrm{H}), 1.60$ (m, $1 \mathrm{H}), 1.45$ (m, $1 \mathrm{H}), 1.21(\mathrm{~s}, 3 \mathrm{H}), 1.16(\mathrm{~s}, 3$ $\mathrm{H}), 1.05-1.10(\mathrm{~m}, 21 \mathrm{H}) \mathrm{ppm} .{ }^{13} \mathrm{C}\left(\mathrm{CDCl}_{3}, 400 \mathrm{~Hz}\right): \delta 136.60,125.54,78.42,73.25,60.55$, 36.82, 29.74, 26.70, 23.47, 18.25, 16.62, 12.26 ppm. FTIR ( $\mathrm{cm}^{-1}$, film): IR: 3393, 2942, 2865, 1463, 1381, 1060, 1012. MS (EI): $367\left(\mathrm{M}+\mathrm{Na}^{+}\right)$. Calculated for $\mathrm{C}_{19} \mathrm{H}_{40} \mathrm{O}_{3} \mathrm{Si}+\mathrm{Na}^{+}: 367.2644$;

Found: 367.2635. The enantiomeric purity was determined by ${ }^{1} \mathrm{H}$ NMR integration of the corresponding mono-MTPA ester derivative $\left(\mathrm{CDCl}_{3}, 500 \mathrm{MHz}\right): \delta 5.24 \mathrm{ppm}(\mathrm{t}, 1 \mathrm{H},(\mathrm{R}$, major)), 5.30 ppm (t, $1 \mathrm{H},(\mathrm{S}$, minor)).

\section{References for Experimental}

(1) Dale, J. A.; Mosher, H. S. J. Org. Chem. 1970, 35, 35, 4002.

(2) Guette, J. P.; Spassky, N.; Boucherot, D. Bull. Soc. Chim. Fr. 1972, 4217.

(3) Wang, Z. -M.; Kolb, H. C.; Sharpless, K. B. J. Org. Chem. 1994, 59, 51104

(4) Berti,, G.; Bottari, F. J. Org. Chem. 1960, 25, 1286.

(5) Döbler, C.; Mehltretter, G. M.; Sundermeier, U.; Beller, M. J. Am. Chem. Soc. 2000, 122, 10289.

(6) Corey, E. J.; Jardine, P. D.; Virgil, S.; Yuen, P. -W.; Connell, R. D. J. Am. Chem. Soc. 1989, $111,9243$.

(7) Heathcock, C. H.; Pirrung, M. C.; Young, S. D.; Hagen, J. P.; Jarvi, E. T.; Badertscher, U.; Märki, H. -P.; Ontgomery, S. H. J. Am. Chem. Soc. 1984, 106, 8161.

(8) Berti, G.; Macchia, F.; Monti, L. J. Chem. Soc. C. 1971, 3371.

(9) 1-Butylcyclohexene was synthesized from cyclohexene oxide: Doris, E.; Dechoux, L.; Mioskowski, C. Tetrahedron Lett. 1994, 35, 7943.

(10) Corey, E. J.; Guzman-Perez, A.; Noe, M. C. J. Am. Chem. Soc. 1995, 117, 10805. 
